\title{
A MATHEMATICAL MODEL OF THE BIOLOGICAL CONTROL OF PREDATORS AND THREE PREY SPECIES
}

\author{
S. MUSA ${ }^{1}$, A. O. ADESANYA ${ }^{1}$, T. A. SHAMAKI',* \\ ${ }^{1}$ Mathematics Department, MAUTECH, Yola, Nigeria \\ ${ }^{2}$ Department of Mathematics, USCOEGA - Yobe State, Nigeria \\ *Corresponding author: stado@mautech.edu.ng \\ Received May 21, 2020
}

Abstract. A modified Leslie - Gower model of predators - preys interaction between three species of prey and a class of predator is considered. Local stability analysis by analytical approach points out that there are number of equilibrium points out of which four significance ones were studied for possible application in biological control. The simulation results illustrated that, even in the absence of ladybug beetles, the swift intra-species competition among aphids, thrips and whiteflies for available recourses is capable of constraining their individual growing population. Accordingly, it is established that, the number of ladybug beetle must be monitored not to reach its full 'carrying capacity' so as to maintain an effectual coexistence between predators and prey species for the purpose of bio-control.

2010 Mathematics Subject Classification. 65L99; 74H20; 74H55; 92B05; 97M60.

Keywords and phrases. Bio-mathematics; Predator-Prey Model; Bio-control and Local Stability.

\subsection{Introduction}

Over the years, modeling the dynamics of biological system by means of differential equations is one of the primary concerns of many applied scientists [1]. It is a wellknown established fact that even though a good number of environmental, engineering, economic and social problems could be reduced to some kind of predator-prey system 
[2]; yet predator - prey equations have its origin in mathematical biology after the fundamental work of Lotka in 1924 and subsequent addition by Volterra in 1926 as rightly reported by Murray [3]. Nevertheless, the study of predator - prey systems are, undoubtedly, far from exhaustive and have, to a large extend, affected studies in many other diverse areas.

For quite a long period of time, the question of coexistence, stability, persistence and extinction as well as predator switching between preys population have been the focus of most researchers, see [4], [5], [6], [7] and the number of references therein. Thus, the ultimate aim of mathematical models of prey - predation edifice and other ecological processes is to provide a basis for the understanding of the dynamics of species populations. But our knowledge of the way predation operates is hardly precise enough as yet to provide a clearer picture of the effects of predation on prey populations. Hence, it is still of interest to apply the Leslie - Gower model in order to see the possibility of how predator specie and three interacting prey species will coexist in an isolated environment for the purpose of biological control.

Jackson and Chen-Charpentier [8] used a mathematical model of Ordinary Differential Equations (ODEs) to model the dynamics of this biological process. They advocated for biocontrol as an alternative solution to insect vectors whose activities typically caused virus propagation in plants. But Jackson [9] mainly considered interaction between the plant hosts, single vector specie, and one class of predator leaving out some essential natural features. This calls for a modification, because under normal circumstances, there may be two, three, or more species of vectors interacting within themselves giving rise to an analogous mathematical model with a number of predators and preys. Therefore, for better understanding, this could be viewed as a sub-model within an ecological environment studied in [9]. 
At the moment, to the best of our knowledge, no work has been carried out on such systems using Leslie - Gower model approach where individual facultative predator specie consumes three distinct species of prey based on preference. Precisely, the key parameters to be accounted include but not limited to the predator's preference to particular prey specie over the others as well as the dynamism of intra-specific competition among prey's species. This is an aspect that Jackson and Chen-Charpentier [8] did not take into cognizance. And more profoundly, most researchers that modified Lotka-Volterra predator - prey model stressed mainly the issue of logistic growth which was originally proposed by Pierre F. Verhulst $(1804-1849)$ as oppose to Malthusian model [10].

In another development, Korobeinikov [11] asserts that "sometime between 1948 and 1958, Leslie introduced a predator - prey model where the 'carrying capacity' of the predator's environment is proportional to the number of prey". As such, the issue of carrying capacity as an arbitrary constant has been dealt with. This is justified by the fact that there are upper limits to the rates of increase in both prey $H$ and predators $P$ population which Leslie - Gower model treated but Jackson and Chen-Charpentier [8] did not take into account. Therefore, this necessitates the introduction of the first Leslie - Gower model [12] given by (1.1) and second Leslie - Gower model [13] given by (1.2) as follows:

$$
\begin{aligned}
& \frac{d H}{d t}=\left(r_{1}-a_{1} P\right) H, \frac{d P}{d t}=\left(r_{2}-a_{2} \frac{P}{H}\right) P \\
& \frac{d H}{d t}=\left(r_{1}-a_{1} P-b_{1} H\right) H, \frac{d P}{d t}=\left(r_{2}-a_{2} \frac{P}{H}\right) P
\end{aligned}
$$

For obvious reason, we shall be considering system (1.2) since it is understood that system (1.1) is a simplification of system (1.2) in which intra specie competition has negligible influences on prey population growth (i.e. $\left.b_{1}=0\right)$. 


\subsection{The Model Formulation}

Generally, the ecological processes of determining the success or otherwise of biological control are multifaceted most especially by considering the diversity of target pests and biological control organisms involved. Therefore, this study considers a scenario where a class of aphids (green peach aphids), thrips (T. tabasi) and whiteflies (Bemisia tabasi) are controlled by natural predatory enemies - ladybugs (hippodamia convergens) for the purpose of biological control. In one hand, it is understood that the interaction between ladybugs beetle and that of vector species (aphids, thrips and whiteflies) is govern by predator - prey principles. On the other hand, due to shortage of available resources, there is swift intra-specific competition among vector species.

We also assumed that the predator population grows logistically with carrying capacity equal to the population size of the preys (aphids, thrips and whiteflies). The biomathematics literatures have many references that justify such type of predators' growth (for example, see Murray [3], May [14], Tanner [15] and Arancibia-Ibarra et al., [17]. We assumed that the intrinsic growth rate should be greater than natural death rate, so that we have a population that grows for all prey species. Again we suppose that the interaction is for short period of time not longer than 120 days (this is due to biological control consideration). This kind of predation has been studied both for its mathematical properties and its efficiency for describing real ecological systems such as mite and spider mite, lynx and hare, sparrow and sparrow hawk by [1] and [15].

We partitioned the vectors population into three compartments based on the order of preference by the predator specie as follows: the number of aphids $A(t)$, the number of thrips $T(t)$ and the number of whiteflies $W(t)$ and we maintain one predator compartment $P(t)$ which preys on all the vectors. Table 1 gives the highlight of the model state variable and parameters. 


\section{Table 1: State Variables and Parameters of the Model}

\begin{tabular}{|c|c|}
\hline Symbol & Descriptions of the State Variable/Parameter \\
\hline$A(t)$ & Number of Aphids at time $t$. \\
\hline$T(t)$ & Number of Thrips at time $t$. \\
\hline$W(t)$ & Number of Whiteflies at time $t$. \\
\hline$P(t)$ & Number of Predators at time $t$. \\
\hline$N(t)$ & Total number of State Variables $N(t)=\mathrm{A}(t)+\mathrm{T}(t)+\mathrm{W}(t)+P(t)$ \\
\hline$a_{1}$ & The capture efficiency rate of predator due to interest on aphids prey \\
\hline$a_{2}$ & The capture efficiency rate of predator due to interest on thrips prey \\
\hline$a_{3}$ & The capture efficiency rate of predator due to interest on whiteflies prey \\
\hline$b_{1}$ & The intra-specific competition rate among aphids prey specie \\
\hline$b_{2}$ & The intra-specific competition rate among thrips prey specie \\
\hline$b_{3}$ & The intra-specific competition rate among whiteflies prey specie \\
\hline$r_{1}$ & The intrinsic growth rate of aphids specie \\
\hline$r_{2}$ & The intrinsic growth rate of thrips specie \\
\hline$r_{3}$ & The intrinsic growth rate of whiteflies specie \\
\hline$r_{4}$ & The intrinsic growth rate of predator (ladybugs) specie \\
\hline$a_{4}$ & The predator's intra-specific competition rate for preys \\
\hline$a_{41}$ & Conversion efficacy (offspring production) of the predator due to aphids \\
\hline$a_{42}$ & Conversion efficacy (offspring production) of the predator due to thrips \\
\hline$a_{43}$ & Conversion efficacy (offspring production) of the predator due to whiteflies \\
\hline$u$ & Natural death rate \\
\hline$t$ & Time \\
\hline
\end{tabular}

Based on the outlined underlining assumptions the model reduces to the following system of non-linear ODEs: 


$$
\begin{aligned}
& \frac{d A}{d t}=\left(r_{1}-u-a_{1} P-b_{1} A\right) A \\
& \frac{d T}{d t}=\left(r_{2}-u-a_{2} P-b_{2} T\right) T \\
& \frac{d W}{d t}=\left(r_{3}-u-a_{3} P-b_{3} W\right) W \\
& \frac{d P}{d t}=P\left(r_{4}-u+a_{41} A+a_{42} T+a_{43} W-a_{4}\left(\frac{P}{A+T+W}\right)\right)
\end{aligned}
$$

\subsection{Analysis of the Model}

In application, it is interesting to consider the solutions that do not change with time. Such solutions are called steady states solutions because they are obtained at equilibrium points or fixed points. It is of interest because the idea of stability analysis establishes the behavior of the trajectories (solution curves) at the equilibrium point. Generally, if an equilibrium point of an ODEs is stable, it follows therefore that any trajectory whose initial value starts close to the equilibrium point will stay close to it for all future time unless unstable.

In order to determine the stability or instability of an equilibrium point(s) of a nonlinear system, the system under consideration has to be linearized by obtaining the Jacobian matrix of the ODEs evaluated at the equilibrium point(s). The consequence of getting real negative eigenvalues or complex number with negative real part establishes the stability of the system. Therefore, investigating the dynamics of interactions of species is simply determining the sign of eigenvalues of linear (linearized) system by investigating the Jacobian matrix at the equilibrium points.

\subsection{Existence of Equilibrium Points}

The equilibrium points of the system of equation (2.1) - (2.4) are the solution of the system solved by equating the RHS of each equation to zero:

$\left(r_{1}-u-a_{1} P-b_{1} A\right) A=0$ 


$$
\begin{aligned}
& \left(r_{2}-u-a_{2} P-b_{2} T\right) T=0 \\
& \left(r_{3}-u-a_{3} P-b_{3} W\right) W=0 \\
& \left(r_{4}-u+a_{41} A+a_{42} T+a_{43} W-a_{4}\left(\frac{P}{A+T+W}\right)\right) P=0
\end{aligned}
$$

The system has a number of equilibrium points, but we shall consider only six steady states due to their biological significance.

$E_{0}=\{\mathrm{A}=\mathrm{T}=\mathrm{W}=\mathrm{P}=0\}$ (Trivial)

$E_{1}=\{A=T=\mathrm{W}=0, P \neq 0\} \quad$ (In this model, this is also trivial, except otherwise $A+T+W \neq 0)$

$$
\begin{aligned}
& E_{2}=\left\{A=\frac{r_{1}-u}{b_{1}}, \mathrm{~T}=\frac{r_{2}-u}{b_{2}}, \mathrm{~W}=\frac{r_{3}-u}{b_{3}}, \mathrm{P}=0\right\} \\
& E_{3}=\left\{A=0, T=0, \mathrm{~W}=\frac{r_{3}-u}{b_{3}}, P=\frac{\mathrm{W}\left(a_{43} W-u+r_{4}\right)}{a_{4}}\right\} \\
& E_{4}=\left\{A=0, T=\frac{r_{2}-u}{b_{2}}, \mathrm{~W}=\frac{r_{3}-u}{b_{3}}, P=\frac{(\mathrm{T}+\mathrm{W})\left(a_{42} T+a_{43} W-u+r_{4}\right)}{a_{4}}\right\} \\
& E_{5}=\left\{A=\frac{r_{1}-u}{b_{1}}, \mathrm{~T}=\frac{r_{2}-u}{b_{2}}, \mathrm{~W}=\frac{r_{3}-u}{b_{3}}, P=\frac{(\mathrm{A}+\mathrm{T}+\mathrm{W})\left(a_{41} A+a_{42} T+a_{43} W-u+r_{4}\right)}{a_{4}}\right\}
\end{aligned}
$$

\subsection{Existence Conditions of the Equilibrium Points}

We have the following existence conditions:

3.2.1 Provided that there is no state variable i.e. it implies neither predator no prey species the trivial equilibrium $E_{0}=\{0,0,0,0\}$ always exist.

3.2.2 The equilibrium point $E_{1}=\left\{0,0,0, K_{0}\right\}$ is not feasible. Physical meaning implies that the predator population cannot survive without preys. This is because the predator specie grows logistically with carrying capacity equal to the population size of the prey. 
3.2.3 The existence of the equilibrium point $E_{2}=\left\{K_{1}, K_{2}, K_{3}, 0\right\}$ is biologically practical. Physical meaning implies, in the absence of the predator population, the three prey species will increase logistically provided that the intrinsic growth rate of the preys is always higher that natural cause of death.

3.2.4 The equilibrium point $E_{3}=\left\{0,0, K_{3}, K_{0}\right\}$ reduced that system into conventional Lotka - Volterra predator - prey model with individual specie of predator and prey. The existence of this equilibrium point guarantees the interaction between predator and prey provided there is, at least, single specie of prey.

3.2.5 The equilibrium point $E_{4}=\left\{0, K_{2}, K_{3}, K_{0}\right\}$ exist. This is the case where there are two classes of prey and a predator population. As in item 3.2.4, the existence of this equilibrium point guarantees the fact that interaction between individual predator specie and two prey species is biologically possible.

3.2.6 The existence of the equilibrium point $E_{5}=\left\{K_{1}, K_{2}, K_{3}, K_{0}\right\}$ gives good reason for this research. This is because the physical meaning implies that there is a unique point at which all the three prey species and the predator population coexist.

In all the six equilibrium points $K_{i}(i=0,1, \ldots, 4)$ are positive constants provided that $r_{i}-u>0$, or $r_{i}>u \quad i=1,2,3,4$.

\subsection{Stability Analysis}

For the stability analysis, we shall focus on the non-trivial equilibrium points, because even if the two trivial equilibrium points are stable, they are not biologically feasible. Thus, the Jacobian matrix of the system $(2.1)-(2.4)$ at any point $J\left(A^{*}, T^{*}, W^{*}, P^{*}\right)$ is given by:

$$
J=\left[\begin{array}{cccc}
r_{1}-u-a_{1} P-2 b_{1} A & 0 & 0 & -a_{1} A \\
0 & r_{2}-u-a_{2} P-b_{2} T & 0 & -a_{2} T \\
0 & 0 & r_{3}-u-a_{3} P-b_{3} W & -a_{3} W \\
\chi_{1} & \chi_{2} & \chi_{3} & \chi_{4}-\frac{a_{41} P}{A+T+W}
\end{array}\right]
$$




$$
\begin{aligned}
\text { where } \chi_{1} & =P\left(a_{41}+\left(\frac{a_{4} P}{(A+T+W)^{2}}\right)\right), \quad \chi_{2}=P\left(a_{42}+\left(\frac{a_{4} P}{(A+T+W)^{2}}\right)\right), \\
\chi_{3} & =P\left(a_{43}+\left(\frac{a_{4} P}{(A+T+W)^{2}}\right)\right), \text { and } \chi_{4}=\left(r_{4}-u\right)+a_{41} A+a_{42} T+a_{43} W
\end{aligned}
$$

\subsubsection{The equilibrium point $E_{2}$ is locally asymptotically stable (LAS).}

Proof. The Jacobian matrix (3.5) evaluated at a point $J_{E_{2}}$ is given by:

$$
J_{\left[E_{2}\right]}=\left[\begin{array}{cccc}
Q_{1} & 0 & 0 & -a_{1} K_{1} \\
0 & Q_{2} & 0 & -a_{2} K_{2} \\
0 & 0 & Q_{3} & -a_{3} K_{3} \\
0 & 0 & 0 & \chi_{4}
\end{array}\right]
$$

Where $Q_{1}=r_{1}-u-a_{1} P-2 b_{1} A, \quad Q_{2}=r_{2}-u-a_{2} P-b_{2} T$ and $Q_{3}=r_{3}-u-a_{3} P-b_{3} W$. Since the Jacobian matrix evaluated at $E_{2}$ is in the form of an upper triangular matrix, it follows therefore that leading diagonal entries of the matrix (3.6) are the eigenvalues.

Consequently, $Q_{1}<0$ provided that $\left(a_{1} P+2 b_{1} A\right)>\left(r_{1}-u\right)$, also $Q_{2}<0$ provided that $\left(a_{2} P+2 b_{2} T\right)>\left(r_{2}-u\right)$, and moreover $Q_{3}<0$ provided that $\left(a_{3} P+2 b_{3} W\right)>\left(r_{3}-u\right)$ and lastly $\chi_{4}<0$ provided that $\left(a_{41} A+a_{42} T+a_{43} W\right)>\left(r_{4}-u\right)$.

In view of the fact that all parameter are positive and the state variables are non negative, it follows therefore that the result is obvious as whenever the above inequalities hold, then $E_{2}$ is LAS as required.

\subsubsection{The equilibrium point $E_{3}$ is locally asymptotically stable (LAS).}

Proof. The Jacobian matrix of the system (2.1) - (2.4) evaluated at the point $E_{3}$ is given by:

$$
J_{\left[E_{3}\right]}=\left[\begin{array}{cccc}
r_{1}-u-a_{1} K_{0} & 0 & 0 & 0 \\
0 & r_{2}-u-a_{2} K_{0} & 0 & 0 \\
0 & 0 & r_{3}-u-a_{3} K_{0}-b_{3} K_{3} & -a_{3} K_{3} \\
\xi_{1} & \xi_{2} & \xi_{3} & \xi_{4}-\frac{a_{41} K_{0}}{K_{3}}
\end{array}\right]
$$


Where $\xi_{1}=K_{0}\left(a_{41}+\frac{a_{4} K_{0}}{K_{3}^{2}}\right), \xi_{2}=K_{0}\left(a_{42}+\frac{a_{4} K_{0}}{K_{3}^{2}}\right), \xi_{3}=K_{0}\left(a_{43}+\frac{a_{4} K_{0}}{K_{3}^{2}}\right)$,

and $\xi_{4}=\left(r_{4}-u\right)+a_{43} K_{3}$

The characteristic polynomial of a matrix (3.7) is:

$$
\left(r_{1}-u-a_{1} K_{0}-\lambda\right)\left|\begin{array}{ccc}
r_{2}-u-a_{2} K_{0}-\lambda & 0 & 0 \\
0 & r_{3}-u-a_{3} K_{0}-b_{3} K_{3}-\lambda & -a_{3} K_{3} \\
\xi_{2} & \zeta_{3} & \xi_{4}-\frac{a_{41} K_{0}}{K_{3}}-\lambda
\end{array}\right|
$$

This could be simplified further as:

$$
\left(r_{1}-u-a_{1} K_{0}-\lambda\right)\left(r_{2}-u-a_{2} K_{0}-\lambda\right)\left|\begin{array}{cc}
r_{3}-u-a_{3} K_{0}-b_{3} K_{3}-\lambda & -a_{3} K_{3} \\
\zeta_{3} & \xi_{4}-\frac{a_{41} K_{0}}{K_{3}}-\lambda
\end{array}\right|
$$

From the first expression $\left(r_{1}-u-a_{1} K_{0}-\lambda\right)$, it is obvious that $\lambda_{1}<0$ if $a_{1} K_{0}>\left(r_{1}+u\right)$ so also is the second expression $\left(r_{2}-u-a_{2} K_{0}-\lambda\right), \quad \lambda_{2}<0 \quad$ whenever $a_{2} K_{0}>\left(r_{2}+u\right)$

The characteristic polynomial is now

$$
\lambda^{2}+\left(u-r_{3}-\xi_{4}+K_{5}\right) \lambda+K_{6}-K_{7}=0
$$

Where

$$
K_{5}=a_{3} K_{0}+b_{3} K_{3}+\frac{a_{41} K_{0}}{K_{K 3}}
$$

$$
\begin{aligned}
& K_{6}=\xi_{4} r_{3}+b_{3} K_{0} a_{41}+\xi_{3} K_{3} a_{3}+\frac{a_{3} K_{0}^{2} a_{41}}{K_{3}}+\frac{u K_{0} a_{41}}{K_{3}} \\
& K_{7}=u \xi_{4}+\xi_{4} K_{0} a_{3}+\xi_{4} K_{3} b_{3}+\frac{r_{3} K_{0} a_{41}}{K_{3}}
\end{aligned}
$$

Solving equation (3.10) for $\lambda$ gives a complex root with real part $\lambda_{3,4}=\left(r_{3}+\xi_{4}\right)-\left(u+K_{5}\right)$ which could be negative whenever $\left(u+K_{5}\right)>\left(r_{3}+\xi_{4}\right)$ as anticipated. Hence the result, $E_{3}$ is LAS. 


\subsubsection{The equilibrium point $E_{4}$ is locally asymptotically stable (LAS).}

Proof. The Jacobian matrix of the system $(2.1)-(2.4)$ evaluated at the point $E_{4}$ is given by:

$$
J=\left[\begin{array}{cccc}
r_{1}-u-a_{1} K_{0} & 0 & 0 & 0 \\
0 & r_{2}-u-a_{2} K_{0}-b_{2} K_{2} & 0 & -a_{2} K_{2} \\
0 & 0 & r_{3}-u-a_{3} K_{0}-b_{3} K_{3} & -a_{3} K_{3} \\
\xi_{5} & \xi_{6} & \xi_{7} & \xi_{8}-\frac{a_{41} K_{0}}{K_{2}+K_{3}}
\end{array}\right]
$$

Where $\xi_{5}=K_{0}\left(a_{41}+\frac{a_{4} K_{0}}{\left(K_{2}+K_{3}\right)^{2}}\right), \quad \xi_{6}=K_{0}\left(a_{42}+\frac{a_{4} K_{0}}{\left(K_{2}+K_{3}\right)^{2}}\right)$,

$$
\xi_{7}=K_{0}\left(a_{43}+\frac{a_{4} K_{0}}{\left(K_{2}+K_{3}\right)^{2}}\right), \text { and } \xi_{8}=\left(r_{4}-u\right)+a_{42} K_{2}+a_{43} K_{3}
$$

By the last column, the characteristic polynomial of a matrix (3.11) is:

$$
\left(\xi_{8}-\frac{a_{41} K_{0}}{K_{2}+K_{3}}-\lambda\right)\left[\begin{array}{ccc}
r_{1}-u-a_{1} K_{0}-\lambda & 0 & 0 \\
0 & r_{2}-u-a_{2} K_{0}-b_{2} K_{2}-\lambda & 0 \\
0 & 0 & r_{3}-u-a_{3} K_{0}-b_{3} K_{3}-\lambda
\end{array}\right]
$$

Since the matrix of (3.12) has non zero entries at the diagonal only; thus, the roots of the characteristic equation which is otherwise known as eigenvalues are simply the product of the diagonal matrix.

$\left(\xi_{8}-\frac{a_{41} K_{0}}{K_{2}+K_{3}}-\lambda\right)\left(r_{1}-u-a_{1} K_{0}-\lambda\right)\left(r_{2}-u-a_{2} K_{0}-b_{2} K_{2}-\lambda\right)\left(r_{3}-u-a_{3} K_{0}-b_{3} K_{3}-\lambda\right)$

Here, for $\lambda_{1}<0$ to be satisfied, $a_{41} K_{0}>\xi_{8}\left(K_{2}+K_{3}\right)$ must hold, so also for $\lambda_{2}<0$ to be satisfied, $a_{1} K_{0}>\left(r_{1}+u\right)$ must hold. In the same line of thought $\lambda_{3}<0$ to be satisfied, $\left(a_{2} K_{0}+b_{2} K_{2}\right)>\left(r_{2}-u\right)$ must hold, and lastly for $\lambda_{4}<0$ to satisfy the expected negative cafeteria, $\left(a_{3} K_{0}+b_{3} K_{3}\right)>\left(r_{3}+u\right)$ must hold.

The result is very clear as whenever the above inequalities hold, then $E_{4}$ is LAS as required. 


\subsubsection{The equilibrium point $E_{5}$ is locally asymptotically stable (LAS).}

Proof. The Jacobian matrix of the system $(2.1)-(2.4)$ at $E_{5}$ is given by:

$$
J=\left[\begin{array}{cccc}
r_{1}-u-a_{1} K_{0}-2 b_{1} K_{1} & 0 & 0 & -a_{1} K_{1} \\
0 & r_{2}-u-a_{2} K_{0}-b_{2} K_{2} & 0 & -a_{2} K_{2} \\
0 & 0 & r_{3}-u-a_{3} K_{0}-b_{3} K_{3} & -a_{3} K_{3} \\
\chi_{5} & \chi_{6} & \chi_{7} & \chi_{8}-\chi_{9}
\end{array}\right]
$$

Where $\chi_{5}=K_{0}\left(a_{41}+\left(\frac{a_{4} K_{0}}{\left(K_{1}+K_{2}+K_{3}\right)^{2}}\right)\right), \chi_{6}=K_{0}\left(a_{42}+\left(\frac{a_{4} K_{0}}{\left(K_{1}+K_{2}+K_{3}\right)^{2}}\right)\right)$,

$$
\begin{aligned}
& \chi_{7}=K_{0}\left(a_{43}+\left(\frac{a_{4} K_{0}}{\left(K_{1}+K_{2}+K_{3}\right)^{2}}\right)\right), \chi_{8}=\left(r_{4}-u\right)+a_{41} K_{1}+a_{42} K_{2}+a_{43} K_{3} \text { and } \\
& \chi_{9}=\frac{a_{41} K_{0}}{K_{1}+K_{2}+K_{3}} .
\end{aligned}
$$

Similarly, manipulating the characteristic polynomial of a matrix (3.13) is by the last column we get:

$$
J=\left(\chi_{8}-\chi_{9}-\lambda\right)\left[\begin{array}{ccc}
r_{1}-u-a_{1} K_{0}-2 b_{1} K_{1}-\lambda & 0 & 0 \\
0 & r_{2}-u-a_{2} K_{0}-b_{2} K_{2}-\lambda & 0 \\
0 & 0 & r_{3}-u-a_{3} K_{0}-b_{3} K_{3}-\lambda
\end{array}\right]
$$

As could be observed, the matrix of (3.14) has non zero entries at the diagonal only; thus, the eigenvalues are simply the product of the diagonal matrix.

$$
\left(\chi_{8}-\chi_{9}-\lambda\right)\left(r_{1}-u-a_{1} K_{0}-2 b_{1} K_{1}-\lambda\right)\left(r_{2}-u-a_{2} K_{0}-b_{2} K_{2}-\lambda\right)\left(r_{3}-u-a_{3} K_{0}-b_{3} K_{3}-\lambda\right)
$$

Once more, for $\lambda_{1}<0$ to be satisfied, $\chi_{9}>\chi_{8}$ is the requirement, so also for $\lambda_{2}<0$ to be satisfied, then it implies that $\left(a_{1} K_{0}+2 b_{1} K_{1}\right)>\left(r_{1}+u\right)$. And finally, the last two eigenvalues are obtained as already presented in sub-section 3.3.4 i.e. for $\lambda_{3}<0$ to be satisfied, $\left(a_{2} K_{0}+b_{2} K_{2}\right)>\left(r_{2}-u\right)$ is established, and lastly $\lambda_{4}<0$ implies $\left(a_{3} K_{0}+b_{3} K_{3}\right)>\left(r_{3}+u\right)$ is true. Therefore, result is very obvious provided the above inequalities hold, then $E_{5}$ is LAS as required. 


\subsection{Numerical Simulation}

Analytical methods are most suitable for theoretical development of mathematics, but unfortunately most mathematical modeling problems, especially the non-linear models have no analytic (closed form) solution. As such, one has no option than to resort to qualitative study of the mathematical properties of the model so as to have clue about the behavior of the solution.

Nevertheless, with the aid of electronic computer (numerical methods) gives approximate solutions, which are almost accurate.

4.1.1 Simulation Result of Three Prey Species in the Absence of Predator Population

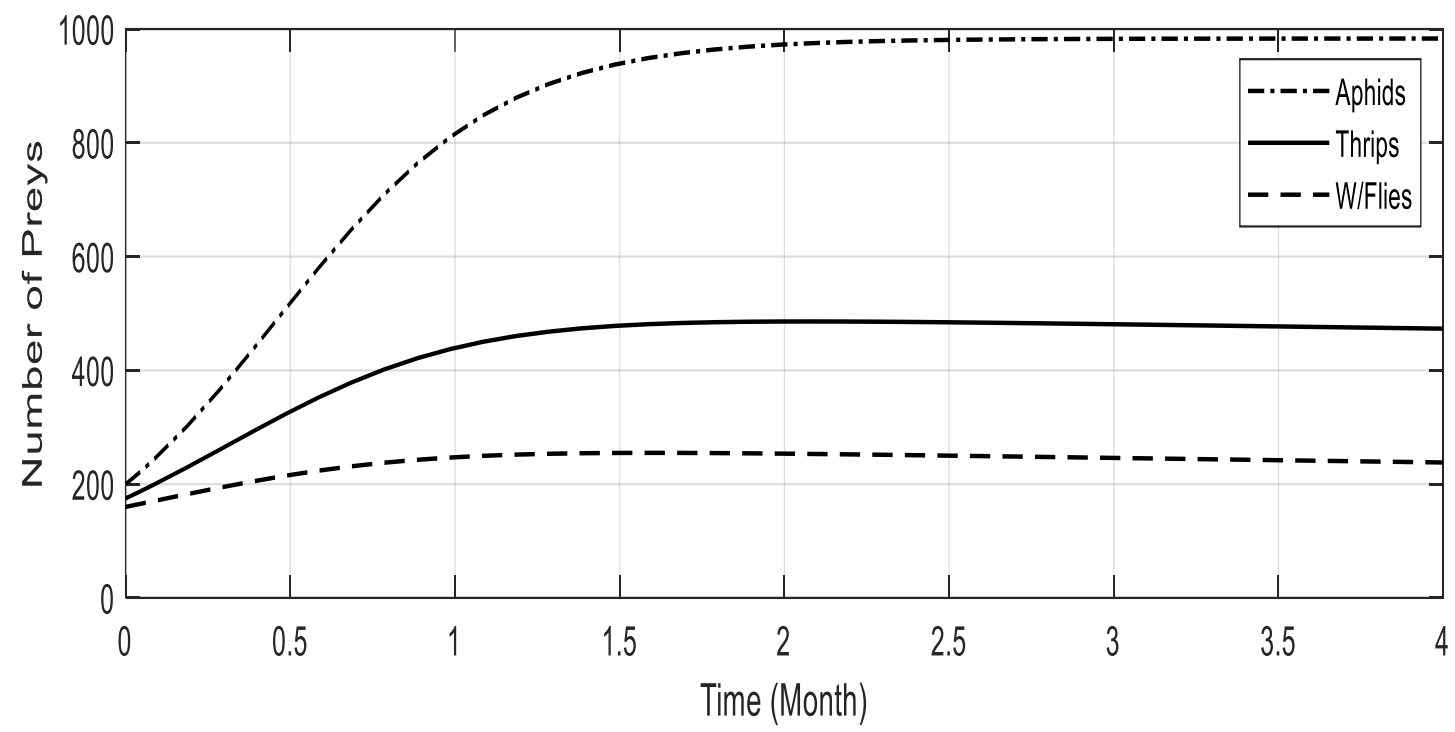

Fig. 4.1: Prey Species in the Absence of Predator

From Fig 4.1 above, it can be observed that the absence of ladybugs beetle will accord aphids, trips and whiteflies the opportunity to grow logistical. As such, the issue of carrying capacity as an arbitrary constant has been dealt with. This means, even in the absence of 'enemy' population, the swift intra-specie competition among vector species for available recourses is capable of controlling the growing size of the 
preys' population. Thus, it is clear then that the modified Leslie - Gower model takes good care of Verhulst's issue of carrying capacity which aimed at resulting into logistic growth model.

4.1.2 Simulation Result of Interaction between One Prey Specie and Predator Population

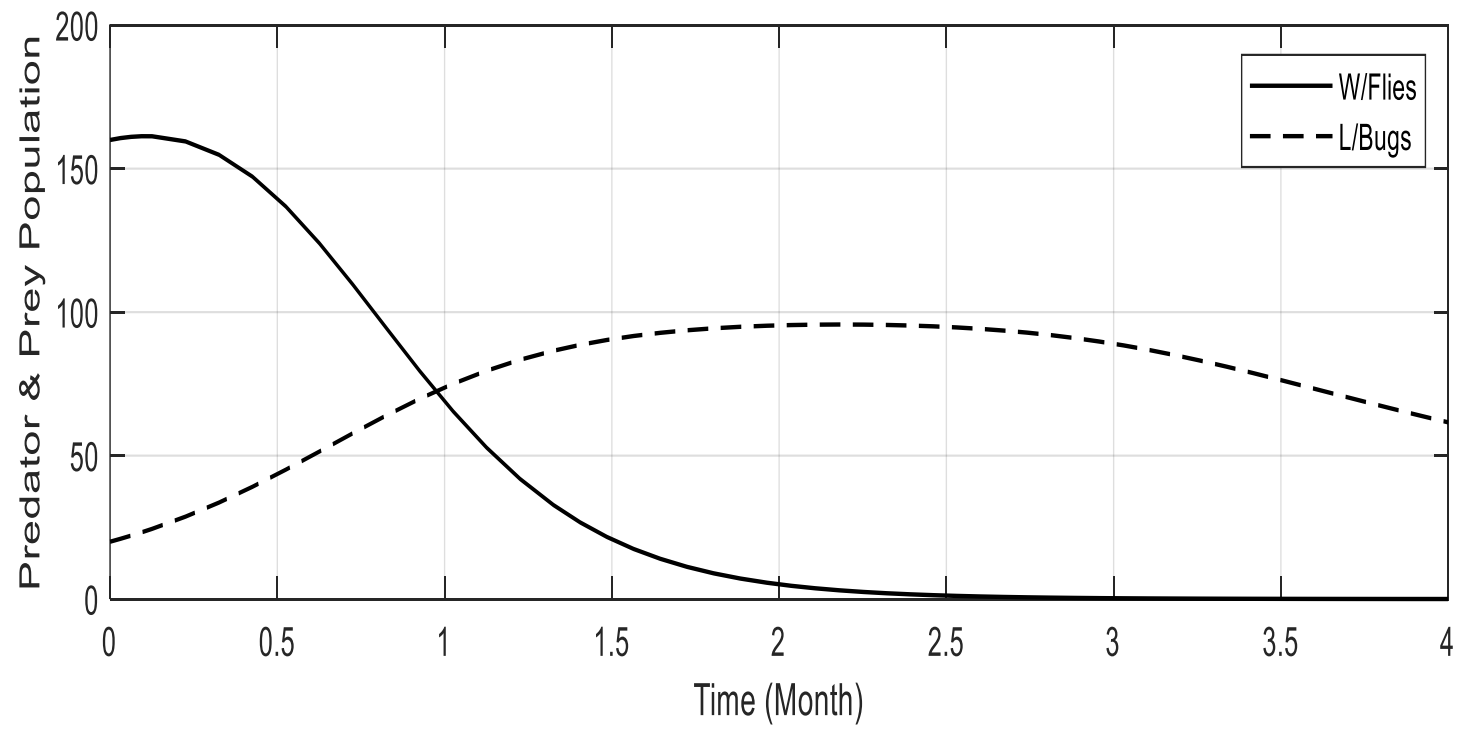

Fig. 4.2: Interaction between Whiteflies and Ladybugs Beetle

In this scenario, the plot in Fig 4.2 concurs with Korobeinikov's assertion [11] that the Leslie model treated predator - prey system where the carrying capacity of the predator's environment is proportional to the number of prey. This has justified the existence of an upper limits to the rates of increase in both whiteflies and ladybugs population which Leslie - Gower model uncovered. Furthermore, due to the effect of predation, the population of whiteflies will go into extinction after two and half months thereby causing the population of ladybug beetles to be reducing due to hunger and starvation. 
4.1.3 Simulation Result of Interaction between Two Prey Species and Predator Population

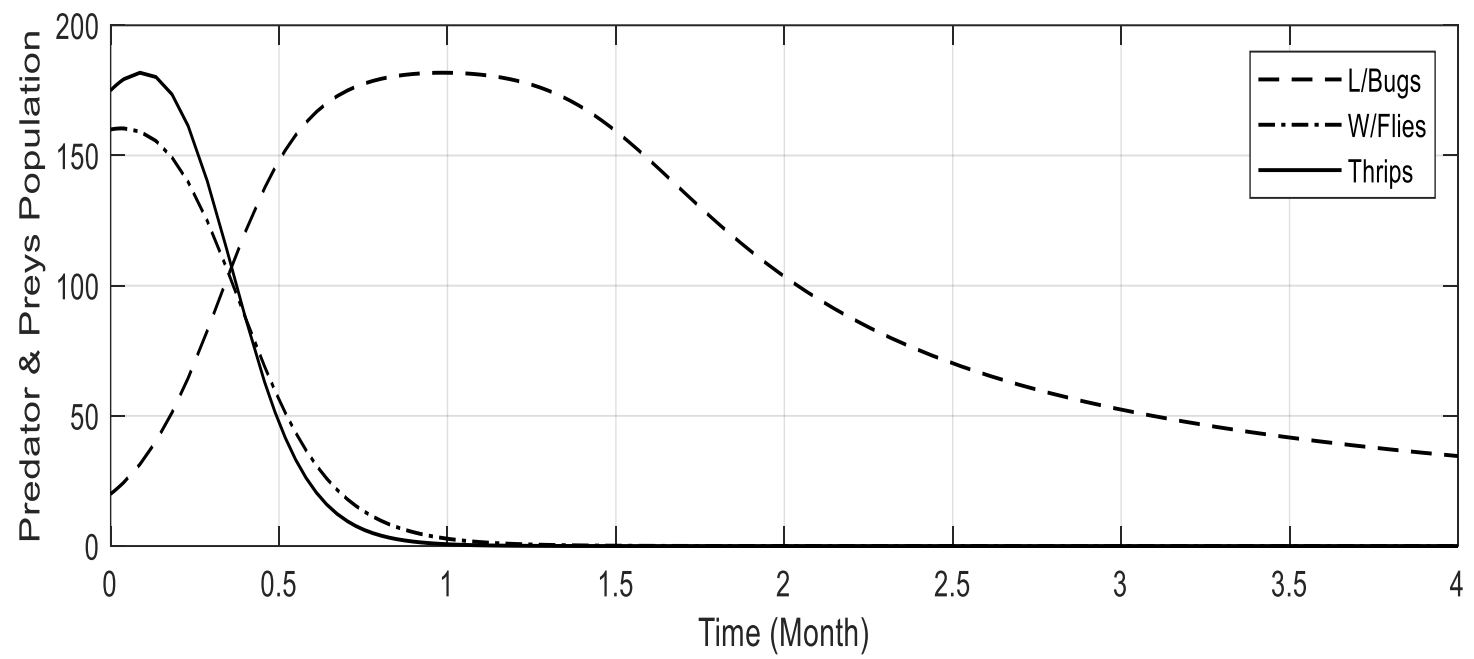

Fig. 4.3: Interaction between Thrips, Whiteflies and Ladybugs Beetle

The plot in Fig. 4.3 advocates that, in a predator - prey mode of interaction, even when the intrinsic growth rate is greater than the natural cause of death, the prey's population will be declining due to predation. But as soon as the predator's population reaches its maximum carrying capacity by means of exhausting all the available vectors, the predator's population cannot be maintained because its own sole means of livelihood is finished; hence, this will cause a corresponding decrease in the predator's population. Therefore, for the purpose of biological control, the number of ladybug beetles must be monitored not to reach its full maximum carrying capacity so as to manage coexistence among all the species involved. 
4.1.4 Simulation Result of Interaction between Three Prey Species and Predator Population

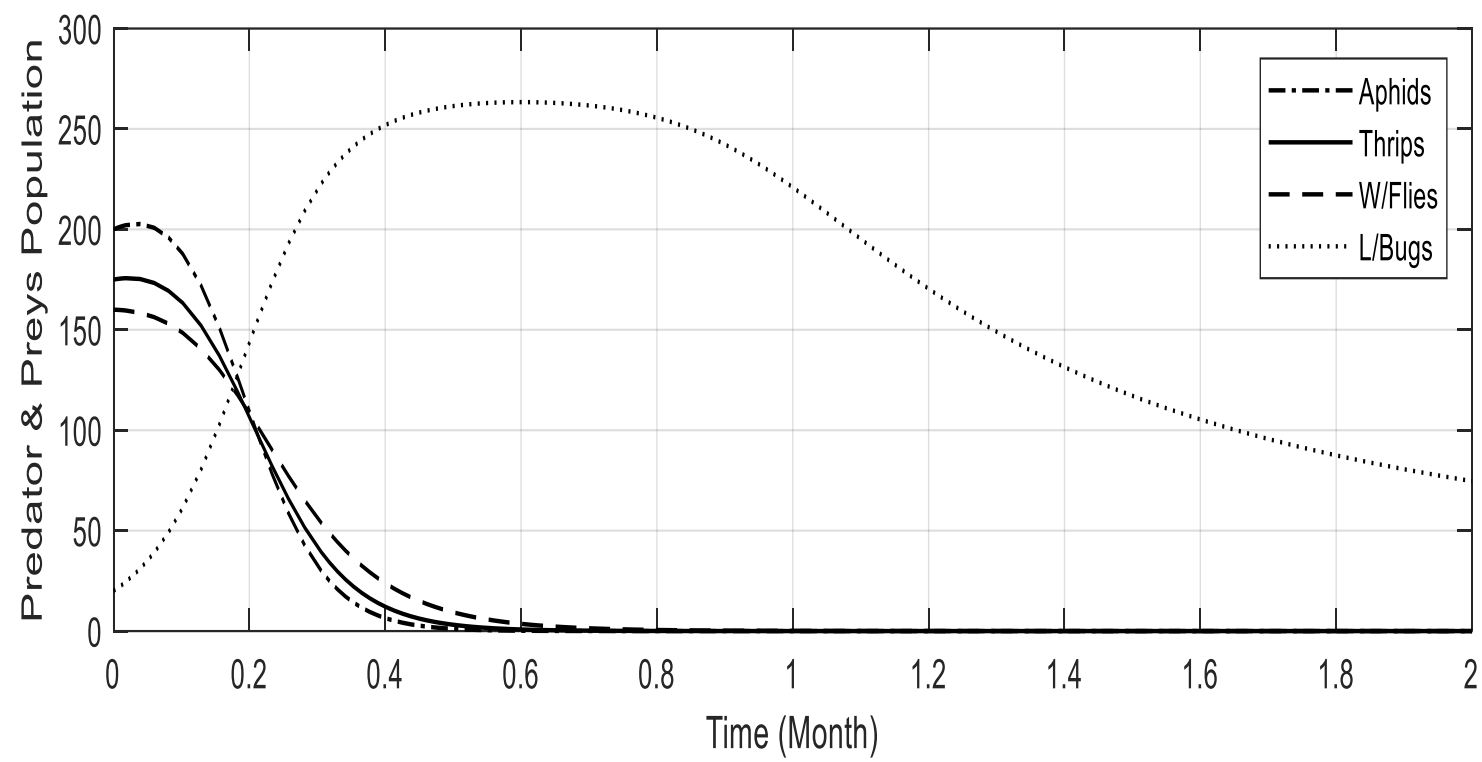

Fig. 4.4: Interaction between Aphids, Thrips, Whiteflies and Ladybugs Beetle As evident from Fig. 4.4 above, it is understood that the interaction between ladybugs beetle and that of three vector species (aphids, thrips and whiteflies) is govern by predator - prey principles. This will cause the vectors' population into extinction based on the scale level of preference of the predator. Interestingly, since the interaction is for short period of time, ladybug beetle will serve as good biological control agent against aphids, thrips and whiteflies. In such case, special care must be taken in order to ensure that the effect of predation do not exterminate all the vectors as their presence is helpful in bio diversity especially for maintenance of the eco system [7]. 


\subsection{Conclusion}

A mathematical model of biological control of predators and three species of prey indicates that, absence of ladybug beetles as predator will give away room for vectors aphids, trips and whiteflies the opportunity to grow logistical not due to their carrying capacity but the result of the swift intra-species competition for available recourses. This concurs with [11] that Leslie - Gower model works in such a manner that the carrying capacity of the predator's environment is proportional to the number of prey. Thus, as demonstrated by the simulation results, as soon as the predators reach their maximum carrying capacity by exhausting all the available vectors, their population will decline. Therefore, for the purpose of biological control, the number of ladybug beetles must be monitored not to reach its maximum carrying capacity so as to have reasonable coexistence among the entire living organisms.

\section{REFERENCES}

[1] D. Wollkind, J. Collings, J. Logan, Metastability in a temperature-dependent model system for predator-prey mite outbreak interactions on fruit trees, Bull. Math. Biol. 50 (1988), 379-409.

[2] X.C. Huang, L. Zhu. Limit cycles in a general Kolmogorov model. Nonlinear Anal., Theory Meth. Appl. 60 (8) (2005), 1393-1414.

[3] J. D. Murray. Mathematical Biology Springer Verlag Berlin. Heidelberg, New York. 1989.

[4] R.P. Canale, An analysis of models describing predator-prey interaction. Biotechnol. Bioeng. 12 (3) (1970), 353-378.

[5] J. Chattopadhyay, S. Pal, A. El Abdllaoui. Classical predator-prey system with infection of prey population—a mathematical model. Math. Meth. Appl. Sci. 26 (14) (2003), 1211-1222.

[6] D. Greenhalgh, M. Haque. A predator-prey model with disease in the prey species only. Math. Meth. Appl. Sci. 30 (8) (2007), 911-929.

[7] M. Rafique, M. Abdul Qader. Population dynamics model for coexistence of three interacting species. Appl. Comput. Math. 4 (4) (2015), 258-263. 
[8] M. Jackson, B.M. Chen-Charpentier, Modeling plant virus propagation with seasonality, J. Comput. Appl. Math. 345 (2019), 310-319.

[9] M. Jackson, Modeling Plant Virus Propagation and an Optimal Control. PhD dissertation, 2018.

[10]M. Peleg, M.G. Corradini, M.D. Normand. The logistic (Verhulst) model for sigmoid microbial growth curves revisited. Food Res. Int. 40 (7) (2007), 808-818.

[11]A. Korobeinikov. A Lyapunov function for Leslie-Gower predator-prey models. Appl. Math. Lett. 14 (6) (2001), 697-699.

[12]P.H. Leslie, Some further notes on the use of matrices in population mathematics. Biometrika 35 (1948), 213-245.

[13]P.H. Leslie, A stochastic model for studying the properties of certain biological systems by numerical methods. Biometrika. 45 (1-2) (1958), 16-31.

[14]R.M. May, Stability and complexity in model ecosystems. Vol. 1. Princeton university press, 2019.

[15]J.T. Tanner, The stability and the intrinsic growth rates of prey and predator populations. Ecology. 56, (4) (1975), 855-867.

[16]C. Arancibia-Ibarra, J.D. Flores, G. Pettet, P. Heijster. A holling-tanner predator-prey model with strong allee effect. Int. J. Bifurcat. Chaos 29 (11) (2019), 1930032. 